\title{
A REMARK ON MEROMORPHIC SOLUTIONS OF DIFFERENTIAL EQUATIONS
}

\author{
SH. STRELITZ
}

\begin{abstract}
The following assertion is proven. Suppose the functions $F, P, Q$ of the differential equation $F\left(z, w, w^{\prime}, w^{(n)}\right)=P(z, w) / Q(z, w)$ to be polynomials of all their corresponding variables. If the considered equation has a transcendental meromorphic solution in $|z|<\infty$, then $Q(z, w)$ does not depend on $w$. An example of possible applications is stated.
\end{abstract}

We would like to recall the following problem which has been widely investigated in the analytic theory of differential equations: to find, if possible, all differential equations of a given form which have solutions of a given class; for example: to find all first order differential equations of the form $y^{\prime}=f(x, y)$, where $f(x, y)$ is a rational function of $x$ and $y$, and every solution of which is a transcendental meromorphic (entire) function (known complete result: the Riccati (linear) equation $y^{\prime}=y^{2}+p(x) y+q(x)\left(y^{\prime}=\right.$ $p(x) y+q(x))$ which alone has the required property [1], [4]).

This note deals with a question of this kind.

We consider the differential equation

$$
F\left(z, w, w^{\prime}, \ldots, w^{(n)}\right)=P(z, w) / Q(z, w),
$$

where $F, P$ and $Q$ are polynomials of all their corresponding variables. Our main result is contained in the following assertion.

Theorem. Suppose the polynomials $P(z, w)$ and $Q(z, w)$ to be mutually prime. If there exists a transcendental meromorphic solution of equation (1) in the finite $z$ plane, then $Q(z, w)$ does not depend on $w$.

We would like to propose an application of this theorem: an equation of the form

$$
w^{(n)}=P(z, w) / Q(z, w)
$$

with polynomials $P$ and $Q$ can have a transcendental meromorphic solution only if $Q(z, w)$ does not depend on $w$, that is, (2) must be of the form

$$
w^{(n)}=P(z, w)
$$

where $P(z, w)$ is a polynomial of $w$ and a rational function of $z$. Very simple considerations show that

Received by the editors November 1, 1976.

AMS (MOS) subject classifications (1970). Primary 34A20, 30A70.

Key words and phrases. Meromorphic solutions, algebraic differential equations.

๑ American Mathematical Society 1977 


$$
w^{(n)}=P(z, w) \equiv \sum_{j=0}^{n+1} a_{j}(z) w^{j}
$$

with rational $a_{j}(z), j=0,1,2, \ldots, n$ (some $a_{j}(z)$ may be identically zero). If we require the existence of a transcendental entire solution of (2) then

$$
w^{(n)}=P(z, w) \equiv \sum_{j=0}^{1} a_{j}(z) w^{j}=a_{1}(z) w+a_{2}(z) .
$$

We omit the corresponding considerations which are almost trivial in the case of a meromorphic solution. In the case of an entire solution the WimanValiron relations [5] (see also (1.2)) can be used.

1. Proof of the Theorem. In the following we use the notation $\theta(x)(\theta$ and $x$ may be complex) for a function defined on a corresponding set $H$ with the property

$$
x^{N} \theta(x) \underset{x \rightarrow \infty, x \in H}{\rightarrow} 0
$$

for every constant real $N$.

Suppose $w(z)$ is a transcendental meromorphic solution of equation (1) in the finite complex plane $|z|<\infty$. The proof of the Theorem is a consequence of the following assertion.

Lemma. Suppose $Q(z, w)$ of (1) depends on $w$. Then there exists an infinite set of points $\{\zeta\}$ with $z=\infty$ as limit point such that

$$
\sigma(\zeta) \equiv P(\zeta, w(\zeta))=\theta(\zeta), \omega(\zeta) \equiv Q(\zeta, w(\zeta))=\theta(\zeta)
$$

We will now show how the proof of the Theorem follows from the Lemma. Let

$$
\begin{array}{ll}
\varphi_{j}(z)=\sum_{n=0}^{\infty} A_{j n} z^{\left(p_{j}-n\right) / q_{j}}, & j=1,2, \ldots, \lambda, \\
\Psi_{j}(z)=\sum_{n=0}^{\infty} B_{j n} z^{\left(t_{j}-n\right) / s_{j}}, & j=1,2, \ldots, \mu,
\end{array}
$$

be correspondingly all the solutions of the algebraic equations $P(z, w)=0$ and $Q(z, w)=0$; here $q_{j}, s_{j}>0, p_{j}$, and $t_{j}$ are integers. In order to solve the equation $P(\zeta, w(\zeta))=\sigma(\zeta)$ we write it in the following form:

$$
\prod_{j=1}^{\lambda}\left(w(\zeta)-\varphi_{j}(\zeta)\right)=\sigma(\zeta)
$$

Then denoting

$$
\left|w(\zeta)-\varphi^{*}(\zeta)\right|=\min _{j=1,2, \ldots, \lambda}\left|w(\zeta)-\varphi_{j}(\zeta)\right|,
$$

we obtain

$$
\left|w(\zeta)-\varphi^{*}(\zeta)\right|^{\lambda} \leqslant|\sigma(\zeta)|,
$$

whence the solution of the considered equation 


$$
w(\zeta)=\varphi^{*}(\zeta)+\theta(\zeta)
$$

where $\varphi^{*}(z)$ coincides with one of the functions $\varphi_{j}(z)$ of $(3.1), j$ depending on $\zeta$. Similarly we also get

$$
w(\zeta)=\Psi^{*}(\zeta)+\theta(\zeta)
$$

where $\Psi^{*}(z)$ is one of the functions $\Psi_{j}(z)$ of $(4.1), j$ depending on $\zeta$, as the solution of the equation $Q(\zeta, w(\zeta))=\omega(\zeta)$. Thus $w(\zeta)$ is the solution of the system (2.1) and therefore

$$
\varphi^{*}(\zeta)-\Psi^{*}(\zeta)=\theta(\zeta)
$$

The number of the functions $\varphi_{j}(z)$ and $\Psi_{i}(z)$ is finite. Consequently, there is an infinite subset of points $\left\{\zeta^{*}\right\} \in\{\zeta\}$ for which $\varphi^{*}(z)$ and $\Psi^{*}(z)$ coincide with two functions $\varphi_{\alpha}(z)$ and $\Psi_{\beta}(z)$, where $\alpha$ and $\beta$ are constant. The equality (8.1) according to (3.1) and (4.1) now gives us

$$
\varphi_{\alpha}\left(\zeta^{*}\right)-\Psi_{\beta}\left(\zeta^{*}\right)=\sum_{n=0}^{\infty}\left(A_{\alpha n} \zeta^{*\left(p_{\alpha}-n\right) / q_{\alpha}}-B_{\beta n} \zeta^{*\left(t_{\beta}-n\right) / s_{\beta}}\right)=\theta\left(\zeta^{*}\right)
$$

In view of the conditions of the Theorem, the polynomials $P(z, w), Q(z, w)$ are mutually prime and thus the system

$$
P(z, w)=0, \quad Q(z, w)=0
$$

has only a finite number of solutions. Hence also the equation

$$
\varphi_{\alpha}(z)-\Psi_{\beta}(z)=0
$$

has only a finite number of solutions and

$$
\varphi_{\alpha}(z)-\Psi_{\beta}(z)=\sum_{n=0}^{\infty} C_{n} z^{(p-n) / q} \not \geq 0,
$$

where $C_{0} \neq 0, p$ and $q$ are certain numbers. From (9.1) now follows

$$
C_{0}=(I+o(I)) \zeta^{*-p / a} \theta(\zeta) \underset{\zeta^{*} \rightarrow 0}{\rightarrow} 0 .
$$

But the last relation is impossible. The contradiction thus obtained proves the Theorem: $Q(z, w)$ does not depend on $w$.

We still have to prove the Lemma. Although, as we will see, the idea of the proof is very simple, it requires relatively many computations which may seem a little boring, but, alas, we do not know any other proof.

In the following we establish the correctness of the Lemma.

2. The proof of the Lemma and thereby, as we have shown in the previous section, of the Theorem, is based on the following simple remark: If $w(z)$ is a transcendental meromorphic solution of (1), then obviously each zero of the function $Q(z, w(z))$ is a zero of $P(z, w(z))$ too. This means that the function $Q(z, w(z))$ has only a finite number of zeros, because according to the conditions of the Theorem the system (10.1) has only a finite number of solutions. Let

$$
Q(z, w) \equiv \prod_{j=1}^{m} Q_{j}(z, w)
$$


be the decomposition of $Q(z, w)$ into irreducible polynomials. Our considerations above show that every $Q_{j}(z, w(z))$ has only a finite number of zeros and therefore for each $Q_{j}(z, w)$ which depends on $w$, we have

$$
Q_{j}(z, w(z))=p_{j}(z) / \eta_{j}(z)
$$

where $p_{j}(z)$ is a polynomial and $\eta_{j}(z)$ is an entire transcendental function. Suppose $Q_{1}(z, w)$ depends on $w$. Denote

$$
p_{1}(z)=p(z), \quad \eta_{1}(z)=\eta(z), \quad p(z) / \eta(z)=\omega^{*}(z) .
$$

We will now show that the set of maximum points $\zeta$ where

$$
|\eta(\zeta)|=\max _{|z|=r}|\eta(z)|=M(r), \quad|\zeta|=r,
$$

is the set $\{\zeta\}$ required by the Lemma. Evidently,

$$
\zeta^{N} p(\zeta) / \eta(\zeta)=\zeta^{N} \omega^{*}(\zeta)=\theta(\zeta),
$$

because $\eta(z)$ is an entire transcendental function and therefore [3]

$$
\lim _{r \rightarrow \infty} \frac{\ln M(r)}{\ln r}=\infty
$$

The solution of the equation

$$
Q_{1}(\zeta, w(\zeta))=\omega^{*}(\zeta)
$$

is given by the function (7.1), where $\Psi^{*}(z)$ is the corresponding function of the set (4.1). Let $Q_{i}(z, w)$ be mutually prime to $Q_{1}(z, w)$, a polynomial of the decomposition (1.2). Then assuming

$$
Q_{i}(z, w)=\sum_{l=0}^{m_{i}} \sum_{k=0}^{n_{i}} a_{k l}^{(i)} z^{l} w^{k},
$$

we get, in view of (4.1), (6.1) and (4.2),

$$
\begin{aligned}
Q_{i}(\zeta, w(\zeta)) & =\sum_{l=0}^{m_{i}} \sum_{k=0}^{n_{i}} a_{k l}^{(i) \zeta^{l}}\left(\Psi^{*}(\zeta)+\theta(\zeta)\right)^{k} \\
& =\sum_{l=0}^{m_{i}} \sum_{k=0}^{n_{i}} a_{k l}^{(i)} \zeta^{l}\left[\Psi^{*}(\zeta)\right]^{k}+\theta(\zeta) \\
& =A_{i}(1+o(1)) \zeta^{\gamma_{i}}+\theta(\zeta),
\end{aligned}
$$

where $A_{i}$ (it can also be $A_{i}=0$ ) and $\gamma_{i}$ are constants. Hence according to (8.2) and (4.2), we get

$$
\begin{aligned}
Q(\zeta, w(\zeta)) & =\prod_{j=1}^{m} Q_{j}(\zeta, w(\zeta)) \\
& =\omega^{*}(\zeta) \prod_{j=2}^{m}\left[(I+o(1)) A_{j} \zeta^{\gamma_{j}}\right]+\theta(\zeta)=\theta(\zeta) .
\end{aligned}
$$


Thus we have established the second equality of (2.1).

3. Definition. A set $E$ of points on the axis $r>0$ is called of finite logarithmic measure (f.l.m.) if $\int_{E} d r / r<\infty$.

In order to complete the proof of the Lemma we still have to establish the first relation of (2.1). We begin with the evaluation of the derivatives $\eta^{(j)}(\zeta)$. The Wiman-Valiron theory (see for example [5]) asserts that the inequalities

$$
\left|\eta^{(j)}(\zeta)\right|<M(r) \ln ^{j(1+\alpha)} M(r), \quad|\zeta|=r, \quad j=1,2, \ldots, p,
$$

hold for every constant $\alpha>0$, with integral $p$ and all $r$ possibly outside a sequence $E(\alpha, p)$ of intervals on the axis $r>0$ of f.l.m. From (6.2) we obtain

$$
\omega^{* \prime}(\zeta)=[p(\zeta) / \eta(\zeta)]^{\prime}=\frac{-p(\zeta)}{\eta^{2}(\zeta)} \eta^{\prime}(\zeta)+\frac{p^{\prime}(\zeta)}{\eta(\zeta)},
$$

and because of (1.3),

$$
\begin{aligned}
\left|\omega^{* \prime}(\zeta)\right| & \leqslant\left|p^{\prime}(\zeta) / \eta(\zeta)\right|+|p(\zeta) / \eta(\zeta)| \ln ^{1+\alpha} M(r) \\
& =\left[\left|p^{\prime}(\zeta) / p(\zeta)\right|+\ln ^{1+\alpha} M(r)\right]|p(\zeta) / \eta(\zeta)|<\frac{C \ln ^{\beta} M(r)}{M(r)} \\
& =\theta(r), \quad r \notin E(\alpha, p),
\end{aligned}
$$

where $C>0$ and $\beta>0$ are certain constants which do not depend on $r$ (we have used here (5.2)).

It is easy to prove successively in the same way as above that

$$
\omega^{*(j)}(\zeta)=\theta(\zeta), \quad|\zeta| \notin E(\alpha, p), \quad j=1,2, \ldots, p .
$$

We omit the details for the sake of brevity.

Our next aim is to evaluate the derivatives $w^{(k)}(\zeta), k=1,2, \ldots, n$. For this purpose we intend to find asymptotic expressions for the partial derivatives $Q_{1 z}^{\prime}(\zeta, w(\zeta))$ and $Q_{1 w}^{\prime}(\zeta, w(\zeta))$. First we remark that, since the polynomial $Q_{1}(z, w)$ is irreducible, the system of equations

$$
Q_{1}(z, w)=0, \quad Q_{1}^{\prime}(z, w)=0
$$

has no more than a finite number of solutions. We now substitute in (7.2) $z=\zeta$ and $w=w(\zeta)=\Psi^{*}(\zeta)+\theta(\zeta)$. Then in view of (4.2) and (6.2), we get

$$
Q_{1}(\zeta, w(\zeta))=\theta(\zeta) .
$$

The same considerations which brought us from (7.2) to (8.2) now show that

$$
\begin{aligned}
& Q_{1 z}^{\prime}(\zeta, w(\zeta))=D_{1}(1+o(1)) \zeta^{\gamma_{1}}+\theta(\zeta), \\
& Q_{1 w}^{\prime}(\zeta, w(\zeta))=D_{2}(1+o(1)) \zeta^{\nu_{2}}+\theta(\zeta),
\end{aligned}
$$

where $\nu_{1}, \nu_{2}, D_{1}$ and $D_{2}$, in general, depend on $\zeta$ but are numbers belonging to a certain finite set which is independent of $\zeta$ (it seems appropriate to recall that $\Psi^{*}(\zeta)$ coincides always with one of the expansions (4.1), which is possibly different for different $\zeta$ ). We will now show that for large enough $\zeta$, $D_{2} \neq 0$. Indeed suppose our proposition is wrong for an infinite sequence 
$\zeta_{n} \rightarrow \infty$. Then $w(\zeta)$ would satisfy the following system of equalities:

$$
Q_{1}\left(\zeta_{n}, w\left(\zeta_{n}\right)\right)=\theta\left(\zeta_{n}\right), \quad Q_{1 w}^{\prime}\left(\zeta_{n}, w\left(\zeta_{n}\right)\right)=\theta\left(\zeta_{n}\right)
$$

But (3.3) has no more than a finite set of solutions and (6.3) is analogous to (2.1). Then the same considerations we used proving the Theorem as a consequence of the Lemma will prove now that the system (6.3) is contradictory too. Thus $D_{2} \neq 0$. From (6.2) and (2.3) it immediately follows that outside the set $E(\alpha, 1)$ of f.l.m.,

$$
\begin{array}{r}
w^{\prime}(\zeta)=\frac{\omega^{\prime}(\zeta)-Q_{1 z}(\zeta, w(\zeta))}{Q_{1 w}^{\prime}(\zeta, w(\zeta))}=\frac{-D_{1}}{D_{2}}(1+o(1)) \zeta^{\nu_{1}-\nu_{2}}+\theta(\zeta), \\
|\zeta| \notin E(\alpha, 1),
\end{array}
$$

whence in view of (4.2) and (5.3), we get

$$
w^{\prime}(\zeta)=L_{1}(1+o(1)) \zeta^{\beta_{1}}+\theta(\zeta)
$$

with some bounded $L_{1}=L_{1}(\zeta)$ and $\beta_{1}=\beta_{1}(\zeta)$. Going on with analogous computations we can find consecutively without difficulty that

$$
\begin{aligned}
w^{(k)}(\zeta)=L_{k}(1+o(1)) \zeta^{\beta_{k}}+\theta(\zeta), & \\
k & =0,1,2, \ldots, n, \quad|\zeta| \notin E(\alpha, n),
\end{aligned}
$$

where $L_{k}$ and $\beta_{k}$ are corresponding numbers.

4. We are now able to complete the proof of the Lemma. Coming back to equation (1) we remember that $F\left(z, w, w^{\prime}, \ldots, w^{(k)}\right)$ is a polynomial. Suppose

$$
\begin{aligned}
& F\left(z, w, w^{\prime}, \ldots, w^{(k)}\right) \\
& \quad \equiv \sum_{j+i_{0}+i_{1}+\cdots+i_{n}=0}^{p} a_{j i_{1} i_{2} \cdots i_{n}} z^{j} w^{i_{0}}\left(w^{\prime}\right)^{i_{1}} \cdots\left(w^{(n)}\right)^{i_{n}} .
\end{aligned}
$$

Then according to (7.3), evidently

$$
\begin{aligned}
& F\left(w(\zeta), w^{\prime}(\zeta), \ldots, w^{(n)}(\zeta)\right) \\
& \quad=\sum_{j+i_{0}+i_{1}+\cdots+i_{n}=0}^{p} a_{j i_{0} i_{1} \cdots i_{n}}(1+o(1)) \zeta^{j} \prod_{k=0}^{n} L_{k} \zeta^{i_{k} \beta_{k}}+\theta(\zeta) \\
& =(1+o(1)) L \zeta^{\beta}+\theta(\zeta), \quad|\zeta| \notin E(\alpha, n),
\end{aligned}
$$

with some bounded $L=L(\zeta)$ and $\beta=\beta(\zeta)$. From (1), (2.4) and (9.2) we finally obtain

$$
\begin{aligned}
P(\zeta, w(\zeta)) & =F\left(\zeta, w(\zeta), w^{\prime}(\zeta), \ldots, w^{(n)}(\zeta)\right) \\
& =\left[(1+o(1)) L \zeta^{\beta}+\theta(\zeta)\right] \theta(\zeta)=\theta(\zeta), \quad|\zeta| \notin E(\alpha, n) .
\end{aligned}
$$

Hence the Lemma, and thereby the Theorem, is proven. 


\section{REFERENCES}

1. S. Bank, On the growth of certain meromorphic solutions of arbitrary second order algebraic differential equations, Proc. Amer. Math. Soc. 25 (1970), 791-797.

2. L. Bieberbach, Theorie der gewöhneichen Differentialgleichungen, Springer, Berlin, 1965, 389 pp.

3. B. Ja. Levin, Distribution of zeros of entire functions, Transl. Math. Monographs, vol. 5, Amer. Math. Soc., Providence, R.I., 1964, 493 pp.; reprinted 1972.

4. Y. Malmquist, Sur les fonctions d'un nombre fini de branches définies par les équations différentielles du premier ordre, Acta Math. 36 (1913), 297-343.

5. G. Valiron, Fonctions analytiques, Presses Univ. de France, Paris, 1954, 236 pp.

Department of Mathematics, University of Haifa, Haifa 31 999, IsRael 